\title{
Ethical Aspects of Personal Science for Persons with Parkinson's Disease: What Happens When Self-Tracking Goes from Selfcare to Publication?
}

\author{
Sara Riggare ${ }^{\mathrm{a}, *}$, Maria Hägglund ${ }^{\mathrm{a}}$, Annelien L. Bredenoord ${ }^{\mathrm{b}}$, Martijn de Groot $^{\mathrm{c}}$ \\ and Bastiaan R. Bloem ${ }^{\mathrm{d}}$ \\ ${ }^{a}$ Uppsala University, Department of Women's and Children's Health, Healthcare Sciences and e-Health, \\ Uppsala, Sweden \\ ${ }^{\mathrm{b}}$ University Medical Center Utrecht, Utrecht University, Department of Medical Humanities, \\ Utrecht, The Netherlands \\ ${ }^{\mathrm{c}}$ Radboud University Medical Centre, Health Innovation Labs, Nijmegen, The Netherlands \\ ${ }^{\mathrm{d}}$ Radboud University Medical Centre, Donders Institute for Brain, Cognition and Behaviour, Department \\ of Neurology, Centre of Expertise for Parkinson \& Movement Disorders, Nijmegen, The Netherlands
}

Accepted 21 May 2021

Pre-press 7 June 2021

\begin{abstract}
Using Parkinson's disease as an exemplary chronic condition, this Commentary discusses ethical aspects of using self-tracking for personal science, as compared to using self-tracking in the context of conducting clinical research on groups of study participants. Conventional group-based clinical research aims to find generalisable answers to clinical or public health questions. The aim of personal science is different: to find meaningful answers that matter first and foremost to an individual with a particular health challenge. In the case of personal science, the researcher and the participant are one and the same, which means that specific ethical issues may arise, such as the need to protect the participant against self-harm. To allow patient-led research in the form of personal science in the Parkinson field to evolve further, the development of a specific ethical framework for self-tracking for personal science is needed.
\end{abstract}

Keywords: Parkinson's disease, self-tracking, ethics, remote monitoring, selfcare, patient empowerment

\section{INTRODUCTION}

Parkinson's disease (PD) is a complex neurodegenerative condition displaying a wide range of motor and non-motor symptoms that are generally challenging to manage using available medical interventions [1]. This recognition has further stimulated the impor-

\footnotetext{
*Correspondence to: Sara Riggare, Uppsala University, Department of Women's and Children's Health, Dag Hammarskjölds väg 14B, 1 tr, 75237 Uppsala, Sweden. Tel.: +46 70 3565104; E-mail: sara.riggare@kbh.uu.se.
}

tant ongoing development towards greater selfcare and patient participation in healthcare [2,3]. Indeed, persons with PD (PwPs) have to manage their condition and treatments on their own for most of the time. Examples include the need to ascertain that medically prescribed interventions are followed adequately, but also the responsibility to implement lifestyle interventions, such as exercise and a healthy diet. Additionally, there is an increasing emphasis on self-tracking, as an important way of detecting relevant disease complications in a timelier manner, also 
to monitor patients more closely in their own home living environment. However, PwPs are mostly left in the dark during this process of selfcare, having to operate largely without suitable tools provided by the healthcare system, and without generally accepted biomarkers that could be monitored to inform their decisions. Consequently, active, engaged, and knowledgeable patients now increasingly take matters into their own hands by using self-tracking to perform research on themselves. Sometimes, these activities challenge the current system for ethical oversight and approval of research [4].

Frameworks for ethical conduct in clinical research have evolved over time, as is apparent from the use of the term "subjects" in an article published in 2000 [5], instead of, as is more common today, "participants". But what happens when individuals move beyond actively participating in clinical research to using empirical methods to improve their own selfcare? What happens when people managing health challenges on a daily basis, also known as patients, make use of the possibilities of the Internet and other technological developments to conduct their own research? To what extent do current ethical frameworks apply to these upcoming practices? Do specific ethical challenges emerge when individuals also intend to disseminate their findings by publishing them in a scientific journal?

Here, we will briefly present the emerging field of personal science and examine some of the main ethical considerations related to the use of self-tracking in personal science as well as in clinical group research. The practice of personal science has similarities to for example the fields of mHealth and citizen science but, as we will demonstrate, also evokes specific ethical challenges. For the discussions in this paper, we will focus on PD as an exemplary chronic condition, but the perspectives offered are likely to also be relevant for the ethical challenges of personal science for a wide range of other chronic diseases.

\section{PERSONAL SCIENCE}

Recently the concept of personal science as a framework for research has been introduced. Personal science has been described as: "the practice of using empirical methods to explore personal questions" [6], "self-directed $N$-of-1 studies" [7], "an interest in collecting data about their own bodies or lives in order to obtain insights into their everyday health or performance" [8]. Based on these key references, personal science is here defined as the practice of exploring personally consequential questions by conducting self-directed $N$-of- 1 studies using a structured empirical approach. This practice is utilised by people with different backgrounds and health statuses, and also applied by people who are confronted with challenges and limitations as a result of chronic and progressive diseases, such as PD.

A key method for collecting data in personal science is self-tracking: "a process of deliberately collecting and structuring observations about one's own life" [6]. The phenomenon is as old as humankind and has emerged broadly and evolved along with the unfolding developments in technology and digitalisation. Its societal impact has been shown in the context of what has been referred to as the Quantified Self movement [6]. The wide availability of sensors, wearable devices and smartphones enables data to be collected about most aspects of our lives, including our health [9]. Of note, although self-tracking can be aided by technology, it can also be done simply using pen and paper [9].

Personal science can include both observational and interventional study designs. The generalisability of the approach can vary; the specific methods used by a single participant (namely measurements, data collection, evaluation, etc.) can potentially be generalisable to other persons dealing with similar health issues. For example, a custom-made app which can successfully track tremor in one particular PwP can likely be extended to other PwPs as well. In contrast, interventions that have a demonstrable effect for one individual still require very careful considerations before applying them to someone else.

Personal science can be practiced at different levels of impact. At the first level, the practice is intended to address issues identified by a given individual and to inform and improve the process of selfcare for just this person. Many of us already perform this type of investigations, for example when using commercially available activity trackers as a tool to be informed about and sometimes even improve physical activity. For personal science of that kind, ethical considerations are largely straightforward, and it will therefore not be the main focus of this paper. In contrast, it is in particular when experiences from personal science projects are publicly disseminated, for example in lay language on social media or in scientific publications, and thereby can lead to other people being influenced, that specific ethical challenges emerge. Personal science that is publicly disseminated has similarities with citizen science. Traditionally the main ethical 
challenges in citizen science have been identified as relating to data quality, data sharing and intellectual property, conflicts of interest, and the risk for exploitation of participants [10]. However, the distinguishing feature of personal science; that the person conducting the research is also the person being studied, sets it apart from most citizen science projects. Personal science projects are also in general of less of a collective nature than citizen science [11].

Examples of scientifically published personal science include Larry Smarr's self-diagnosis of inflammatory bowel disease from gut microbiome analyses [12] and Dana Lewis' work in type 1 diabetes, aiming to help both herself and the wider community by developing tools and methods to achieve improved blood glucose control [13]. It has been suggested that patients using personal science in collaboration with clinicians are in a better position to sustain a behavioural change [14].

In summary, the goal of personal science is not merely to collect data but rather to use self-collected data to achieve personally consequential insights that can be used for taking action in relation to a specific issue, often health related. Personal science is not intended to replace clinical research but rather to complement and enrich its practices and improve relevance to individual patients.

\section{Personal science in $P D$}

The practice of personal science has similarities to clinical N-of-1 studies, which have been used in PD by clinicians to study individuals with PD [15-17]. The key difference is that personal science is selfdirected, meaning that the person conducting the study is also the person being studied. To the best of our knowledge, the only peer-reviewed academic work on personal science in PD has been conducted by the first author of this paper (SR); two single subject studies where SR used herself as the research participant $[18,19]$. The first study [18] was conducted with an observational design, exploring how the effects of SR's medication for PD, prescribed by her neurologist, varied across the day with time and with each medication intake. The medication effect was quantified by capturing finger tapping performance with a smartphone app. The second study [19] was conducted with a placebo-controlled interventional design, examining the effect of nicotine from an e-cigarette on levodopa-induced dyskinesias. In both studies, SR used the knowledge she gained to better understand her own personal condition and to improve treatment decisions, both with and without clinical support. In the following, the two personal science studies by SR will be used to inform discussions around ethical aspect of personal science.

\section{ETHICAL ASPECTS OF USING SELF-TRACKING FOR SCIENTIFIC INQUIRY IN PD}

Group research is currently the cornerstone for implementing novel interventions into our healthcare systems and forms the basis for clinical guidelines and protocols and self-tracking as a method for data collection can be used also in that context. Wearable devices and other types of technology are proving to be useful tools for collecting data for research into PD at a group level, for example in studies using smartphone apps [20,21] or smartwatches [22], either alone or in combination with advanced clinical biomarkers, allowing for "deep phenotyping" [23].

However, the direct applicability of group research results to individual patients is limited and many of the personal questions that PwPs have cannot be answered by group research. Examples of such unanswered questions that can be consequential on an individual level include: "How do I respond to this particular drug?"; "How can I time my medications to obtain the best possible effect?", "How can I find the best balance between functionality and medication side effects?", or "Do I sleep better when I exercise more?" This is where personal science can provide benefit.

In fact, the present discussion about personal science raises an almost philosophical issue about science in general, namely that the purpose of all research should ultimately be to benefit not the groups that were studied in a particular study, but rather individuals living with a chronic condition like PD. All too often, research findings are interpreted at the group level, without a sufficient understanding of the possible benefits (or harms) for the participating individuals. This issue is becoming all the more important as we are beginning to realise that PD is not a single condition with a single pathophysiology, but that it may be more appropriate to speak of 7 million different types of parkinsonism, namely as many as there are individuals living with this condition in the world [24]. And that we may ultimately need just as many personalised treatment approaches. The concept of personal science brings this approach a step closer to reality. 
Of course, one could have a discussion about the adequacy of the term personal science, but that is beyond the scope of this paper. For our purposes it is enough to note that personal science can be seen as principally different from clinical research. We will reflect on these differences by elaborating on some important ethical considerations of using self-tracking for clinical and personal science respectively, focused on the topics: self-tracking data, burden of tracking, relevance of research, independent review and dissemination, and protection and fair treatment of participants.

\section{Self-tracking data}

When self-tracking is used for data collection in clinical research, the ethical responsibility lies with the clinician/researcher instructing the patient to collect data. When it comes to using data from digital tools in clinical research, clinical researchers are responsible for making sure that the privacy of the individuals generating the data is protected and potential risks mitigated [25]. As research into mHealth demonstrates, specific ethical issues can arise relating to patients' access to data, data ownership, privacy and security, and the potential exposure of bystanders [26].

Similarly, if digital tools are used to acquire data in personal science, privacy aspects can be an important issue. When individual patients use commercially available tools to track their own disease, there is an inherent risk that these health-related data may be exploited by private companies for their own purposes, such as targeted health advertisements. Of concern is also that such poorly protected health information finds its way to, e.g., insurance companies, who may ultimately hold this against the participant by offering them a less attractive healthcare insurance policy. For individual patients, these long-term consequences are often not immediately apparent, and it may be more difficult for an individual to ascertain the privacy and security aspects when data are acquired with a particular commercially provided device. Such issues need to be addressed, including the question who can be held accountable for the potential risks of personal health data being handled by commercial tech companies.

In the two personal science studies by SR, data were collected using an app that saved data locally on the phone [18], as well as using pen and paper $[18,19]$. This demonstrates that even though ethics relating to self-tracking data can be an issue, per- sonal science in PD can also be done without saving potentially sensitive data online.

\section{Burden of tracking}

Self-tracking can add a significant workload to the already demanding work of being a patient. In clinical group research, clinicians are obliged to minimise potential harm due to the intervention, and to make sure that any potential benefits outweigh the risk of harm to each individual participant [5]. Self-tracking may usefully alleviate the pressure on clinicians or researchers, but it certainly does not come "for free", as participants pay a price with their time investment, as demonstrated in previous research [27].

The aspect of added workload from self-tracking is especially important in PD, given the decreased energy levels and challenges with task management associated with PD. The two personal science studies in PD by SR specifically highlights this added burden of tracking $[18,19]$.

\section{Relevance of research}

The questions explored in research have to be scientifically relevant while not exploiting the participants [5]. This balance may be especially difficult to navigate in clinical group research for PD, since the time to potential benefit for both the individual PwP participating in the clinical group research as well as to the wider PwP community so far has largely failed to keep up with the speed of disease progression within an individual PwP. Furthermore, the evidence is increasing that research priorities, as identified by PwP, often differ from priorities expressed by clinicians and researchers [28-30]. For example, clinicians tend to prioritise motor symptoms and other visible/quantifiable signs of $\mathrm{PD}$, whereas many $\mathrm{PwP}$ lend greatest value to the less visible non-motor symptoms.

It is also worth noting that effects at the individual level can easily be lost at the group level perspective. For example, there are examples of drugs that have been approved based on research in groups that were dominated by men, even though in daily clinical practice, women prove to be much less responsive. Women may also experience significant side effects that were not seen in the study population dominated by men that participated in the original seeding trials. Such differences have also been observed in PD [31]. 
To address such issues, individualised research design, such as personal science, can provide benefit. From a relevance perspective, research into what PwP themselves consider important, using personal science, should be supported. Personal science has the potential to lead to insights that can inform further, more conventional systematic research and may thereby be able to contribute to improving the relevance of research. In personal science, the methodology (measurements, data collection, evaluation etc) is more likely to be generalisable, than the results. For example, in a highly variable and individualised condition like PD, an intervention that works for one PwP might be unsuitable for another, for example because the efficacy or the risk of side effects can differ widely across different individuals. Being cautiously explicit about the limitations of the generalisability is a key element in sharing the results, which was done in the two articles by SR $[18,19]$.

\section{Independent review and dissemination}

Independent review is important for ethical research to ensure that a researcher's conflicting interests do not cause problems, for example in the form of lower quality research [5]. In the US, independent review of clinical research is operationalised by for example granting agencies, local institutional review boards, and data and safety monitoring boards while other countries have other protective mechanisms. The structures for independent review can address different parts of the research process like study design, recruitment of participants etc. The peer reviewers and the journals' editors during the dissemination process can also be considered a form of independent review. For clinical research, wellknown procedures and safeguards are in place for all these phases.

For personal science the situation is currently unclear. Naturally, personal science practiced at the first level of impact, where the individual has the purpose to improve his/her own selfcare, is largely unproblematic. It is in particular when personal science is publicly disseminated that specific ethical challenges emerge. In situations like that, the transferability of the work conducted holds ethical implications since then the work can also have an effect beyond the individual performing the inquiry on themselves.

It has been argued that research led by patients requires adaptations of current ethical standards [32]. Should a person performing personal science with the intention to publish their findings somehow be protected from possibly harming themselves? This is an area where independent review could play an important role. A study of a group practicing personal science explored a process for joint ethical reflections and also present some suggested ethical principles, including transparency, participant control of data and ongoing risk-to-benefit evaluation [33]. We consider this among the most pressing issues regarding ethics of personal science; to explore appropriate mechanisms for independent review of personal science projects. Questions that need addressing include: How can independent review of personal science be implemented in a constructive manner so that new knowledge can be developed and disseminated without risk to personal scientists? At what stage is it reasonable to introduce mechanisms for protecting themselves from self-harm? How should the issue of informed consent be handled in personal science projects? Who should be responsible for deciding about the balance between safety and possible efficacy, as a regular ethics committee would normally do for group research? This area will require further work.

We have examined the two personal science studies in PD by SR with the issue of potential risk for self-harm in mind. For the observational study [18], the risk for self-harm can be considered low, since no other intervention than the medications prescribed by SR's neurologist was introduced. The interventional study [19] deserves more ethical attention, since it involves a self-chosen, non-medical intervention in the form of an e-cigarette. However, this specific intervention should be considered as being associated with a minimal additional risk, also in relation to the potential side effects that conventional medications for PD can entail.

When personal science projects are published in conventional scientific journals, established procedures apply, for example regarding ethical requirements. When SR's two studies [18, 19] were published it was explicitly stated in the manuscripts that the studies had not been reviewed by an ethical review board and a description was given as to how ethical aspects had been taken into account in conducting the study. Both studies were published after conventional review.

\section{Protection and fair treatment of research participants}

In clinical research studies selection of participants has to be done in a fair manner. This includes, e.g., 
Table 1

Summary of key points and future work

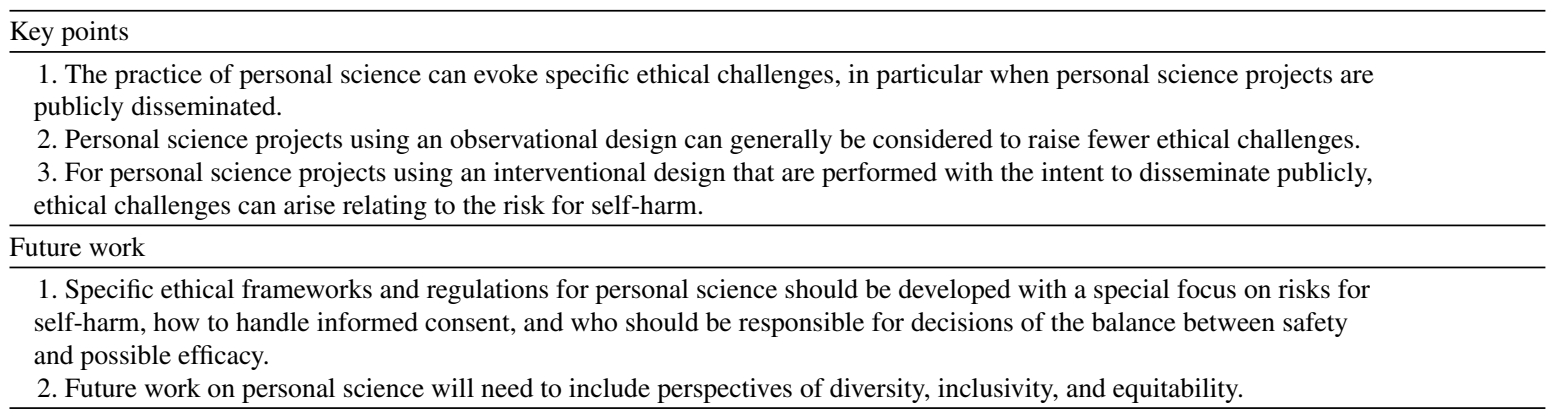

decisions on inclusion and exclusion criteria, recruitment strategies, study site selection, and populations to study [5]. The main difference between conventional clinical research and personal science is in the locus of control. Clinical research is conducted by clinicians on healthy participants and patients. Although conditions have improved as the terminology has evolved from subjects to participants, the fact remains that patients are typically not in control of the research process. Of course, a research participant has the right to discontinue their participation at any time, but then they will also miss out on any potential clinical benefits.

In personal science, the researcher and the participant are one and the same, and the primary goal for launching the personal science study is most often an explicit aim to gain a personal benefit, and this is a marked contrast to traditional group science. This means that in personal science, the participant/researcher is fully in control of the research process and can thereby decide in every stage of the project, if an invested effort is likely to yield sufficient benefits. These potential benefits can also lead to ethical challenges. For example, the desire to alleviate symptoms may motivate an individual to downplay the expected risks or effort associated with a certain intervention, which goes back to the discussion on protection against self-harm in the previous section.

It is also important from a value perspective that resources are used in a fair and just way. In general, patients doing personal science are individuals with high levels of autonomy [27]. They can pave the way for other patients but from an inclusivity perspective, it is important to realise that this route is not open for all. In further work on personal science, we must ensure that individuals and groups that are presently unable to engage in personal science for health, social, economic, or other reasons are not disadvantaged.

\section{CONCLUSIONS AND FUTURE WORK}

We conclude that current ethical requirements that are commonly applied to clinical group research, are not per se suitable for research conducted by PwPs using personal science and that there is a need for development of adapted ethical procedures. To allow patient-led research in the form of personal science in PD to evolve further, specific ethical frameworks and regulations for self-tracking for personal science should be developed. The potential risk for self-inflicted harm should be given specific attention. For a person wanting to engage in personal science projects, ethical aspects always have to be considered. In general, observational designs can be considered unproblematic. When personal science projects intended to be publicly disseminated use interventional design however, specific ethical challenges can arise, which may warrant independent ethical review. More work is needed in this field. A summary of key points and suggested future work is given in Table 1.

\section{ACKNOWLEDGMENTS}

We want to thank Marina Noordegraaf, Jakob Eg Larsen, and Gary Wolf for valuable comments on earlier versions of the article.

\section{CONFLICT OF INTEREST}

The authors have no conflicts of interest to report.

\section{REFERENCES}

[1] Okun MS (2017) Management of Parkinson Disease in 2017: Personalized approaches for patient-specific needs. JAMA 318, 791-792. 
[2] Dorsey ER, Vlaanderen FP, Engelen LJ, Kieburtz K, Zhu W, Biglan KM, Faber MJ, Bloem BR (2016) Moving Parkinson care to the home. Mov Disord 31, 1258-1262.

[3] Hulbert SM, Goodwin VA (2020) 'Mind the gap' - a scoping review of long term, physical, self-management in Parkinson's. Physiotherapy 107, 88-99.

[4] Wicks P (2018) Patient, study thyself. BMC Med 16, 10-12.

[5] Emanuel EJ, Wendler D, Grady C (2000) What makes clinical research ethical? JAMA 283, 2701-2711.

[6] Groot M De, Wolf GI (2020) A conceptual framework for personal science. Front Comput Sci 2, 1-5.

[7] Groot M De, Drangsholt M, Martin-Sanchez FJ, Wolf G (2017) Single subject (N-of-1) research design, data processing, and personal science. Methods Inf Med 6, 416-418.

[8] Heyen NB (2016) Self-tracking as knowledge production: Quantified self between prosumption and citizen science. In Lifelogging: Digital self-tracking and lifelogging - between disruptive technology and cultural transformation, Springer.

[9] Fox S, Duggan M (2013) Tracking for Health. Pew Research Center, https://www.pewresearch.org/internet/ 2013/01/28/tracking-for-health/

[10] Resnik DB, Elliott KC, Miller AK (2015) A framework for addressing ethical issues in citizen science. Environ Sci Policy 54, 475-481.

[11] Wiggins A, Wilbanks J (2019) The rise of citizen science in health and biomedical research. Am J Bioeth 19, 3-14.

[12] Smarr L, Hyde ER, Mcdonald D, Sandborn WJ (2017) Tracking human gut microbiome changes resulting from a colonoscopy. Methods Inf Med 56, 442-447.

[13] Lewis D, Leibrand S (2016) Real-world use of open source artificial pancreas systems. J Diabetes Sci Technol 10, 1411.

[14] Alemi F, Moore S, Baghi H (2008) Self-experiments and analytical relapse prevention. Qual Manag Health Care 17, 53-65.

[15] Rascol O, Ferreira J, Nègre-Pages L, Perez-Lloret S, Lacomblez L, Galitzky M, Lemarié JC, Corvol JC, Brotchie JM, Bossi L (2012) A proof-of-concept, randomized, placebo-controlled, multiple cross-overs (n-of-1) study of naftazone in Parkinson's disease. Fundam Clin Pharmacol 26, 557-564.

[16] Ferreira JJ, Mestre T, Guedes LC, Coelho M, Rosa MM, Santos AT, Barra M, Sampaio C, Rascol O (2016) Espresso coffee for the treatment of somnolence in Parkinson's disease: Results of n-of-1 trials. Front Neurol 7, 27.

[17] van der Velden RMJ, Mulders AEP, Drukker M, Kuijf ML, Leentjens AFG (2018) Network analysis of symptoms in a Parkinson patient using experience sampling data: An $n=1$ study. Mov Disord 33, 1938-1944.

[18] Riggare S, Hägglund M (2018) Precision medicine in Parkinson's disease - exploring patient-initiated selftracking. J Parkinsons Dis 8, 441-446.

[19] Riggare S, Unruh KT, Sturr J, Domingos J, Stamford JA, Svenningsson P, Hägglund M (2017) Patient-driven N-of-1 in Parkinson's disease: Lessons learned from a placebocontrolled study of the effect of nicotine on dyskinesia. Methods Inf Med 56, 123-128.

[20] Bot BM, Suver C, Neto EC, Kellen M, Klein A, Bare C, Doerr M, Pratap A, Wilbanks J, Dorsey ER, Friend SH, Trister AD (2016) The mPower study, Parkinson disease mobile data collected using ResearchKit. Sci Data 3, 160011.
[21] Zhan A, Mohan S, Tarolli C, Schneider RB, Adams JL, Sharma S, Elson MJ, Spear KL, Glidden AM, Little MA, Terzis A, Ray Dorsey E, Saria S (2018) Using smartphones and machine learning to quantify Parkinson disease severity the mobile Parkinson disease score. JAMA Neurol 75, 876-880.

[22] Bloem BR, Marks WJ, Silva De Lima AL, Kuijf ML, Van Laar T, Jacobs BPF, Verbeek MM, Helmich RC, Van De Warrenburg BP, Evers LJW, Inthout J, Van De Zande T, Snyder TM, Kapur R, Meinders MJ (2019) The Personalized Parkinson Project: Examining disease progression through broad biomarkers in early Parkinson's disease. BMC Neurol 19, 160.

[23] Dorsey ER, Omberg L, Waddell E, Adams JL, Adams R, Ali MR, Amodeo K, Arky A, Augustine EF, Dinesh K, Hoque ME, Glidden AM, Jensen-Roberts S, Kabelac Z, Katabi D, Kieburtz K, Kinel DR, Little MA, Lizarraga KJ, Myers T, Riggare S, Rosero SZ, Saria S, Schifitto G, Schneider RB, Sharma G, Shoulson I, Stevenson EA, Tarolli CG, Luo J, McDermott MP (2020) Deep phenotyping of Parkinson's disease. J Parkinsons Dis 10, 855-873.

[24] Bloem BR, Okun MS, Klein C (2021) Parkinson's disease. Lancet, doi: 10.1016/S0140-6736(21)00218-X.

[25] (2016) International Ethical Guidelines for Health-related Research Involving Humans.

[26] Cvrkel T (2018) The ethics of mHealth: Moving forward. $J$ Dent 74, S15-S20.

[27] Riggare S, Duncan TS, Hvitfeldt H, Hägglund M (2019) "You have to know why you're doing this": A mixed methods study of the benefits and burdens of self-tracking in Parkinson's disease. BMC Med Inform Decis Mak 7, 175.

[28] Sjödahl Hammarlund C, Nilsson MH, Idvall M, Rosas SR, Hagell P (2014) Conceptualizing and prioritizing clinical trial outcomes from the perspectives of people with Parkinson's disease versus health care professionals: A concept mapping study. Qual Life Res 23, 1687-1700.

[29] Nisenzon AN, Robinson ME, Bowers D, Banou E, Malaty I, Okun MS (2011) Measurement of patient-centered outcomes in Parkinson's disease: What do patients really want from their treatment? Parkinsonsim Relat Disord 17, 89-94.

[30] Hattori N, Fujimoto K, Kondo T, Murata M, Stacy M (2012) Patient perspectives on Parkinson's disease therapy in Japan and the United States: Results of two patient surveys. Patient Relat Outcome Meas 3, 31-38.

[31] Göttgens I, van Halteren AD, de Vries NM, Meinders MJ, Ben-Shlomo Y, Bloem BR, Darweesh SKL, OerteltPrigione S (2020) The impact of sex and gender on the multidisciplinary management of care for persons with Parkinson's disease. Front Neurol 11, 576121.

[32] Vayena E, Brownsword R, Edwards SJ, Greshake B, Kahn JP, Ladher N, Montgomery J, Connor DO, Neill OO, Richards MP, Rid A, Sheehan M, Wicks P, Tasioulas J (2016) Research led by participants: A new social contract for a new kind of research. J Med Ethics 42, 216-219.

[33] Grant AD, Wolf GI, Nebeker C (2019) Approaches to governance of participant-led research: A qualitative case study. BMJ Open 9, e025633. 